\title{
An Update on Health-Related Quality of Life and Patient-Reported Outcomes in Hidradenitis Suppurativa
}

This article was published in the following Dove Press journal: Patient Related Outcome Measures

\section{J Mac Mahon \\ S Kirthi \\ N Byrne \\ C O'Grady \\ AM Tobin}

Department of Dermatology, Tallaght University Hospital, Tallaght, Dublin 24

Trinity College Dublin, Dublin, Ireland
Correspondence: AM Tobin

Department of Dermatology, Tallaght

University Hospital, Dublin, Ireland

Email atobin@tcd.ie

\begin{abstract}
Hidradenitis suppurativa is a common inflammatory skin condition which causes recurrent abscesses, sinuses and scarring in the axillae, groin and inframammary areas. As well as causing significant physical distress due to pain and discharge, the condition impacts psychological well-being with markedly impaired quality of life. Patients suffer pain, embarrassment and psychological distress with impairment of their work and intimate relationships marking it as one of the most distressing dermatological conditions. Numerous studies have documented markers of psychological distress encompassing the physical effects such as pain and itch, affects on mood and impaired function.
\end{abstract}

Keywords: hidradenitis suppurativa, health-related quality of life, patient-reported outcomes

\section{Introduction}

Adalimumab is a fully humanized monoclonal antibody to TNF-alpha and is licensed for the treatment of moderate to severe hidradenitis suppurativa. In two phase III studies; Pioneer I and II; patients who achieved a clinical response reported clinically meaningful improvement in Dermatology Life Quality Index (60.5\% vs 30.4\%), Pain Numeric Rating Scale (46.9\% vs 19.9\%), hidradenitis suppurativa quality of life (49.4\% vs $26.9 \%$ ), work-related performance (52.6\% vs $37.7 \%$ ), and non-workrelated performance $(59.5 \%$ vs $33.3 \%)$.

Hidradenitis Suppurativa (HS), is a chronic, recurrent and debilitating skin condition affecting the axilla and inguinal areas. Starting as painful nodules it can lead to abscess formation and scarring with significant disfigurement. It often produces a malodorous and recurrent discharge and can cause significant pain and distress particularly during a "flare up". Hidradenitis suppurativa may have a severe impact on the quality of life of patients affected by it mainly due to the nature of the condition, the areas that it affects as well as the relative paucity of effective treatments. In Qualitative studies of patients with HS, feelings of shame, isolation and stigmatization are described, hence it is unsurprising that this condition significantly impacts quality of life. ${ }^{1,2}$ The location of lesions in genital areas can impact imtimate relationships while the pain of the condition as well as the unpredictability of flare-ups may affect a patient's ability to work. In addition to clinical measurements of disease severity, it is important to assess impact on patient's quality of life in Hidradenitis suppurativa. Although there is an increase in research in HS there is still a lack well-defined, robust patient-reported outcome 
measures and Quality of Life is not always measured. These deficits have been recognized in a recent position statement of the European Academy of Dermatology and Venereology. ${ }^{3}$

\section{Health-Related Quality of Life}

Health-related quality of life (HRQoL) is a multidimensional concept which refers to the perception of the overall effects of a disease and its impact on the patients' daily functioning. Various instruments are used to measure HRQoL in dermatology. These include the Skindex-29 and Dermatology Life Quality Index, DLQI) and general (EQ-5D) HRQoL.

\section{DLQI}

The Dermatology Life Quality Index was developed by Finlay et al and is a self-administered 10-item questionnaire, designed to assess the impact of a particular skin condition in the previous week. ${ }^{4}$ Each question is graded $0-3$, with 0 implying no impact and 3 indicating a major impact, the highest score possible is 30 . The 10 questions are divided into 5 domains: symptoms, daily activities, leisure, work/school, personal relationships and impact of treatment. A total score of 2-5 indicates little effect, 6-10 a moderate effect, 11-20 a large effect and a score of 21-30 an extremely large effect on patient's quality of life. ${ }^{5}$ The DLQI has limitations in that it is very much designed as a snap-shot at one timepoint and fails to compute cumulative life impairment. It is still the most widely used instrument and allows comparison across, different skin disease, different treatments and clinical trials.

\section{Skindex-29}

The Skindex-29 is a revised version of an earlier tool which contained 61 questions developed in 1996 and 1997 repsectively. ${ }^{6}$ It was deigned to allow capture of life impairment over time, a shortcoming of the DLQI. It is divided into three domains, symptoms ( 7 items), emotions (10 items) and functioning (12 items). Patients score how often (Never, Rarely, Sometimes, Often, All the time) over the previous four weeks the they experienced a feeling or symptom. Answers are converted to a linear scale 0-100 and generates three scale scores ie the average scores of items within a domain are combined into a domain score.

\section{EQ-5D}

The EuroQol-5D is a generic instrument developed by the European Quality of Group. It consists of a descriptive scale addressing: mobility, self-care, usual activities, pain/ discomfort and anxiety/depression and a visual analog scale (VAS). Two versions currently exist: the EQ-5D-5L with five levels of severity for each question and the EQ5D-3L with three levels of severity. EQ-5D scores range between -0.594 and 1.000 (full health). The instrument has been validated across several different diseases and a number of different languages.

\section{Hospital-Based Studies of HR-Qol in Hidradenitis}

The burden of HS has been investigated in several hospitalbased studies, showing considerable impairment. In a study of 805 Danish patients attending an outpatient clinic, Jemec and colleagues found DLQI, Body Mass Index and smoking had a significant impact on EurQol VAS, Major Depression Inventory (MDI) and Euro-QoL-5D. Significant predictors for the model were DLQI $(\mathrm{P}<0.05)$, BMI points above 25 and active smoking (both: $\mathrm{P}<0.01)$ and $\mathrm{MDI}(\mathrm{P}<0.001)$. Van de Worth and Jemec found 110 patients attending a hospitalbased setting to have a DLQI of $8.9 \pm 8.3^{7}$ In 211 patients attending a Dutch hospital clinic, patients with HS had significantly higher DLQI scores compared to dermatology control patients $8.4 \pm 7.5$ vs $4.3 \pm 5.6(\mathrm{P}<0.0001)$ and higher scores on the MDI Questionnaire 11.0 vs $7.2(\mathrm{P}<0.0001) .{ }^{8}$ Kurek et al confirmed this finding in 90 patients attending a German Clinic where $38.6 \%$ of patients with HS reported depression versus $2.4 \%$ of age, sex and BMI-matched controls. ${ }^{9}$

In a cross-sectional study of 421 Danish patients with HS attending an outpatient dermatology department, patients had significantly decreased EQ-5D index and VAS scores in all age groups except for $65-74$ year olds. ${ }^{10}$ The total index score in the cohort was $85 \%$ of that of the general population (0.705 compared with the population mean of 0.887 ) and the VAS score was $75 \%$ (62.25 compared with 82.6 ), indicating significant disability. Pain and discomfort had the most significant impact on the index. Vinding et al (16) found a mean EQ-5D index of 0.82 (95\% confidence interval (CI): 0.74-0.90 ) and a mean VAS score of 79.56 (95\% CI: 72.89-86.22). It is speculated that the participants had less severe disease than those in the present hospital-based study because they were drawn from the general population. This is supported by the greater disutility found by Matusiak et al, in which 54 hospital-based Polish patients with HS (28 women, 26 men) with a mean age of $39.94 \pm 11.63$ years, completed the EQ-5D questionnaire. ${ }^{11}$ The authors found a mean $\pm \mathrm{SD}$ index and VAS score of $0.66 \pm 0.23$ and $56.78 \pm 18.84$, respectively.

In 94 Greek patients recruited at the dermatology department, the DLQI mean score was $11.43 \pm 6.61$ in patients with 
HS. ${ }^{12}$ The patients with HS presented statistically significantly higher anxiety $(6.41 \pm 3.31$ vs $5.00 \pm 1.59, \mathrm{p}<$ $0.001)$, depression $(5.45 \pm 2.79$ vs $4.16 \pm 1.54, \mathrm{p}<0.001)$ as measured by the Hospital Anxiety and Depression Score (HADS), and loneliness and social isolation scores (42.86 \pm 8.63 vs $35.57 \pm 6.17, \mathrm{p}<0.001)$ as measured by the UCLA Loneliness Scale and lower self-esteem scores (18.91 \pm 1.79 vs $19.77 \pm 2.53, \mathrm{p}=0.008$ ) as measured by the Rosenberg Self Esteem Scale, than healthy controls.

Hazvami et al analyzed HRQoL in five studies based in Pain VAS, DLQI, EuroQOL 5D VAS, Short Form-36, Total Work Productivity Impairment. ${ }^{13}$ Compared with patients with psoriasis, patients with HS reported higher scores for VAS-pain (54.3 vs 36.1 [P $<0.0001])$, Dermatology Life Quality Index (15.3 vs $11.3[\mathrm{P}<0.0001])$, EuroQOL 5D VAS (58.8 vs $50.8[\mathrm{P}<0.0002])$, and Total Work Productivity Impairment (35.4 vs 18.2). Patients with HS had lower Short Form-36 Health Survey scores than did patients with psoriasis (physical, 39.6 vs 49.0; mental, 41.5 vs 47.5 [both $\mathrm{P}<0.0001$ ]).

In a two-centre study of 154 HS patients based in the US and Denmark, $35.7 \%$ of patients were depressed and average DLQI was 10, resilience as measured using the Brief Resilient Coping Scale, appeared to moderate depression in this study. ${ }^{14}$ Among 152 Greek patients with HS, the mean DLQI was 11.7 with patients with Hurley Stage III disease having significantly higher DLQI. ${ }^{15}$ In 52 Canadian patients with HS, those with higher self-reported malodour had significantly higher SkinDex scores but not DLQI scores. There was no difference in mean DLQI scores for the low- vs highodour groups, but patients with high odour had a greater quality of life impairment as measured by the Skindex tool $(\mathrm{t}=-4.19, \mathrm{df}=43, \mathrm{P}<0.0001$, mean difference $=-18.87) .{ }^{16}$

In an interesting probe of the impact of HS on body image researchers in Germany using the Frankfurt Body Concept Scale found that HS significantly reduced body image (mean FKKS score, 234.2 [5.4] in patients and 276.9 [5.7] in controls; $P<0.001$ ), even when controlled for BMI. ${ }^{17}$ A correlation was found for the extent of body image disruption and BMI $(r=-0.589 ; P<0.001)$, HADSdepression score $(r=-0.619 ; P<0.001)$, and HADSanxiety score $(r=-0.340 ; P=0.03)$.

\section{Population-Based Studies of HR-QoL in Hidradenitis Suppurativa}

In a population-based study in Denmark, patients with HS had higher DLQI scores than controls and other patients with psoriasis, eczema or "pimples. ${ }^{18}$ In 55 patients attending community dermatology clinics in Canada. The mean DLQI score was $10 \pm 8.8$, indicating a moderate effect on patients" lives. The authors also assessed the Short Form - 36 version 2 questionnaire in these patients, SF-36v2 scores were significantly reduced with respect to both physical and mental health. The severity of disease, as measured by Hurley staging, the number of lesions, and patient-reported QoL were significantly correlated with the DLQI score $(\beta=0.549,0.285,0.390$, respectively; $\mathrm{p}=$ $0.000,0.045,0.004$, respectively; $\alpha=0.05) .{ }^{19}$

\section{Health -Related Quality of Life and Sexual Health and Sleep}

Three hundred patients with HS showed diminished QoL and sexual health (Female Sexual Function Index: 21·6 $\pm 9 \cdot 6$, International Index of Erectile Function: $49 \cdot 7 \pm 20 \cdot 7$, Arizona Sexual Experience Scale: $16 \cdot 7 \pm 5 \cdot 3$, Dermatology Life Quality Index: $12 \cdot 5 \pm 7 \cdot 5) .{ }^{20}$ Sexual health was associated with QoL in women but not in men. Female sex and late onset of HS were associated with poor sexual function. Impairment of QoL was associated with anogenital involvement, early onset of HS, disease severity and disease activity. In a study of 3845 dermatology patients in 13 countries, patients with HS had the highest rates of sexual impairment as measured using the DLQI. ${ }^{21}$ When compared with the male control group, male patients with HS had on average a lower sexual SQoLM $(\mathrm{p}<0.0001)$ as measured by the Sexual Quality of Life Questionnaire for Use in Men (SQoLM) and International Index of Erectile Dysfunction (IIEF) $(\mathrm{p}<0.019) .{ }^{21}$ Female patients had significantly higher distress related to sexual function as measured with the Female Sexual Distress Scale - Revised (FSDS-R). FSDS$R$ compared with healthy female volunteers $(p=0.002)$.

In recent work from Poland by Kaaz et al, patients with HS had poorer sleep quality than controls as measured by Pittsburgh Sleep Quality Index (PSQI). The mean scores for PSQI were $6.5 \pm 3.6$ points (range $0-18$ ) and $3.1 \pm 1.9$ points (range $0-7$ ) for patients with HS and control subjects, respectively $(\mathrm{p}<0.001){ }^{22}$ When considering insomnia as measured using the Athens Insomnia Scale there was no difference. Itch and pain were associated with poorer sleep quality.

\section{Hidradenitis-Suppurativa Quality of Life: HS-QOL}

The above studies and myriad of measures has led to an attempt at standardizing measures of Quality of Life in HS 
using a disease-specific measure the HS-QOL. This was developed to assimilate the three domains of skin-related QOL, impact on general well-being and psychosocial impairment and initially consisted of a 53-item questionnaire. $^{23}$ The HS QOL was validated in 55 patients with HS in four different centres and showed good concordance. $^{24}$ The HS-QoL was reduced from 53 items to 44 items, resulting in a 7-subscale questionnaire. All subscales demonstrated excellent internal consistency, except for the support subscale, which had adequate internal consistency. All 7 HS-QoL subscales were related to other measures of QoL, life satisfaction, and mental health, which demonstrates convergent validity.

\section{Adalimumab and HRQoL in Hidradenitis Suppurativa}

Adalimumab is the first licensed treatment for HS and the first indication that it impacted quality of life in patients with HS came from case-reports and small case series. In 6 Spanish patients treated with Adalimumab $40 \mathrm{mg}$ fortnightly increasing to weekly, Bianco et al recorded significant reduction in DLQI 1 month and 1 year after commencing treatment. ${ }^{24}$ Amano et al also reported significant reduction in DLQI in 10 patients treated with Adalimumab $160 \mathrm{mg} \mathrm{s} / \mathrm{c}$ week, $80 \mathrm{mg}$ week 1 and $40 \mathrm{mg}$ alternate weeks thereafter. ${ }^{25}$ In the next prospective study of adalimumab using the same dosage regimen, Miller et al reported a significant reduction in 21 patients with $\mathrm{HS}^{26}$ In another prospective open-label study of the use of adalimumab $80 \mathrm{mg}$ week 1 then $40 \mathrm{mg}$ fortnightly for 24 weeks, 15 patients with moderate to severe HS experience significant drop in DLQI scores, scores began to rise again by week $48 .^{27}$

There has been one Phase II parallel, randomized, placebo-controlled trial consisting of a blinded 16-week period (period 1) and an open-label 36-week period (period 2) of adalimumab administered in outpatient clinics in the US of adalimumab. ${ }^{28,29}$ Two Phase III trials, PIONEER I and II have also been conducted ${ }^{30-32}$ Kimball A and colleagues carried out a post-hoc analysis of the pooled data from Pioneer I and II studies to evaluate the impact of Hidradenitis Suppurativa Clinical Response (HiSCR) on PROM. ${ }^{33}$ Pooling placebo and active treatment arms, 39\% of patients (245/629) achieved HiSCR at week 12. This included DLQI, Patients Global Assessment of Skin Pain Numeric Rating Scale (0-11), HS Quality of Life Numeric Rating Scale (0-11), The Work Productivity and Activity Impairment (WPAI) questionnaire, a validated instrument that evaluates 4 areas: work time missed because of HS (absenteeism), impairment while working because of HS (presenteeism), overall work impairment because of HS, and impairment of daily activities because of HS (activity impairment). The Treatment Satisfaction Questionnaire for Medication (TSQM) which includes assessments of the patient's satisfaction with a medication's effectiveness, lack of side effects, convenience, and global satisfaction with the medication. The TSQM is scaled from 0 to 100 points, with lower scores indicative of a greater dissatisfaction. Irrespective of treatment, significantly ( $p$ $<0.05$ ) more HiSCR responders than non-responders experienced clinically meaningful improvement in Dermatology Life Quality Index (60.5\% vs 30.4\%), Pain Numeric Rating Scale $(46.9 \%$ vs $19.9 \%)$, hidradenitis suppurativa quality of life (49.4\% vs $26.9 \%)$, work-related performance $(52.6 \%$ vs $37.7 \%)$, and non-work-related performance $(59.5 \%$ vs $33.3 \%$ ).

Patient-reported outcomes and HR-Qol are probably more important in hidradenitis suppurativa than many other skin conditions. Although the use of multiple measures makes comparison across studies difficult there is consensus regarding the need for comprehensive measures of quality of life in HS and a move towards standardization. Adalimumab is the first treatment licensed for use in HS and has shown good results in DLQI, HsQol, pain, work-related and non-work-related performance.

It is evident that HS confers a considerable psychological burden on patients. The heterogeneity of patient reported outcomes applied to different inflammatory diseases precludes comparison with other skin diseases such as eczema and psoriasis. In a study from our clinic, we compared DLQI and HADS in patients with HS and patients with psoriasis (personal communication). Patients with HS had significantly higher scores in DLQI compared to patients with psoriasis (12.2 vs 9.9. p < 0.001). This was significant across all domains of the DLQI: daily activities, work, leisure activities, personal relationships and treatment. HS patients had significantly higher mean depression score on the HADS, 7.3 vs 4.2 in the psoriasis group, $\mathrm{p}<0.001$ and higher anxiety scores 9.6 vs $7.7(p<0.005)$. Assessment of psychological distress is embedded in treatment algorithms of psoriasis and this must be replicated in HS.

\section{Disclosure}

Professor Anne-Marie Tobin reports grants from Abbvie, during the conduct of the study. The authors report no conflicts of interest in this work. 


\section{References}

1. Kirby JS. Qualitative study shows disease damage matters to patients with hidradenitis suppurativa. J Am Acad Dermatol. 2016;74 (6):1269-1270. doi:10.1016/j.jaad.2016.01.001

2. Keary E, Hevey D, Tobin AM. A qualitative analysis of psychological distress in Hidradenitis Suppurativa.mBr. J Dermatol. 2019. doi:10.1111/bjd. 18135

3. Chernyshov PV, Zouboulis CC, Tomas-Aragones L, et al. Quality of life measurement in hidradenitis suppurativa: position statement of the European Academy of Dermatology and Venereology task forces on quality of life and patient-oriented outcomes and acne, rosacea and hidradenitis suppurativa. J Eur Acad Dermatol Venereol. 2019.

4. Finlay AY, Khan GK. Dermatology Life Quality Index (DLQI)-a simple practical measure for routine clinical use. Clin Exp Dermatol. 1994;19(3):210-216. doi:10.1111/ced.1994.19.issue-3

5. Lewis V, Finlay AY. 10 years experience of the Dermatology Life Quality Index (DLQI). J Investig Dermatol Symp Proc. 2004;9 (2):169-180. doi:10.1111/j.1087-0024.2004.09113.x

6. Chren MM, Lasek RJ, Quinn LM, Mostow EN, Zyzanski SJ. Skindex, a quality-of-life measure for patients with skin disease: reliability, validity, and responsiveness. $J$ Invest Dermatol. 1996;107(5):707-713. doi:10.1111/1523-1747.ep12365600

7. von der Werth JM, Jemec GB. Morbidity in patients with hidradenitis suppurativa. Br J Dermatol. 2001;144:809-913. doi:10.1046/j.13652133.2001.04137.x

8. Onderdijk AJ, van der Zee HH, Esmann S, et al. Depression in patients with hidradenitis suppurativa. $J$ Eur Acad Dermatol Venereol. 2013;27(4):473-478. doi:10.1111/jdv.2013.27.issue-4

9. Kurek A, Johanne Peters EM, Sabat R, Sterry W, Schneider-Burrus S. Depression is a frequent co-morbidity in patients with acne inversa. J Dtsch Dermatol Ges. 2013;11(8):743-9, 743-50. doi:10.1111/ddg. 12067

10. Riis PT, Vinding GR, Ring HC, Jemec GB. Disutility in patients with hidradenitis suppurativa: a cross-sectional study using EuroQoL-5D. Acta Derm Venereol. 2016;96(2):222-226. doi:10.2340/00015555-2129

11. Matusiak L, Bieniek A, Szepietowski C. Psychophysical aspects of hidradenitis suppurativa. Acta Derm Venereol. 2010;90:264-268. doi:10.2340/00015555-0866

12. Kouris A, Platsidaki E, Christodoulou C, et al. Quality of life and psychosocial implications in patients with hidradenitis suppurativa. Dermatology. 2016;232(6):687-691. doi:10.1159/000453355

13. Hamzavi IH, Sundaram M, Nicholson C, et al. Uncovering burden disparity: a comparative analysis of the impact of moderate-to-severe psoriasis and hidradenitis suppurativa. J Am Acad Dermatol. 2017;77 (6):1038-1104. doi:10.1016/j.jaad.2017.07.027

14. Kirby JS, Butt M, Esmann S. Jemec GBE association of resilience with depression and health-related quality of life for patients with hidradenitis suppurativa. JAMA DERMATOL. 2017;153 (12):1263-1269. doi:10.1001/jamadermatol.2017.3596

15. Katoulis AC, Liakou AI, Rotsiamis N, et al. Descriptive epidemiology of hidradenitis suppurativa in Greece: a study of 152 cases. Skin Appendage Disord. 2017;3(4):197-201. doi:10.1159/000475822

16. Alavi A, Farzanfar D, Lee RK, Almutairi D. The contribution of malodour in quality of life of patients with hidradenitis suppurativa. J Cutan Med Surg. 2018;22(2):166-174. doi:10.1177/120347541 7745826

17. Schneider-Burrus S, Jost A, Peters EMJ, Witte-Haendel E, Sterry W, Sabat R. Association of hidradenitis suppurativa with body image. JAMA DERMATOL. 2018;154(4):447-451. doi:10.1001/jamadermatol. 2017.6058
18. Vinding GR, Knudsen KM, Ellervik C, Olesen AB, Jemec GB. Selfreported skin morbidities and health-related quality of life: a population-based nested case-control study. Dermatology. 2014;228 (3):261-268. doi:10.1159/000358009

19. Alavi A, Anooshirvani N, Kim WB, Coutts P, Sibbald RG. Quality-of -life impairment in patients with hidradenitis suppurativa: a Canadian study. Am J Clin Dermatol. 2015;16(1):61-65. doi:10.1007/s40257014-0105-5

20. Janse IC, Deckers IE, van der Maten AD, et al. Sexual health and quality of life are impaired in hidradenitis suppurativa: a multicentre cross-sectional study. $B r \quad J$ Dermatol. 2017;176(4):1042-1047. doi:10.1111/bjd.14975

21. Sampogna F, Abeni D, Gieler U, et al. Impairment of sexual life in 3485 dermatological outpatients from a multicentre study in 13 European countries. Acta Derm Venereol. 2017;97(4):478-482. doi:1 $0.2340 / 00015555-2561$

22. Kaaz K, Szepietowski JC, Matusiak Ł. Influence of itch and pain on sleep quality in patients with hidradenitis suppurativa. Acta Derm Venereol. 2018;98(8):757-761. doi:10.2340/00015555-2967

23. Sisic M, Kirby JS, Boyal S, Plant L, McLellan C, Tan J. Development of a quality-of-life measure for hidradenitis suppurativa. J Cutan Med Surg. 2017;21(2):152-155. doi:10.1177/12 03475416677721

24. McLellan C, Sisic M, Oon HH, Tan J. Preliminary validation of the HS-QoL: a quality-of-life measure for Hidradenitis suppurativa. J Cutan Med Surg. 2018;22(2):142-146. doi:10.1177/1203475417736281

25. Blanco R, Martínez-Taboada VM, Villa I, et al. Long-term successful adalimumab therapy in severe hidradenitis suppurativa. Arch Dermatol. 2009;145(5):580-584. doi:10.1001/archdermatol.2009.49

26. Amano M, Grant A, Kerdel FA. A prospective open-label clinical trial of adalimumab for the treatment of hidradenitis suppurativa. Int J Dermatol. 2010;49(8):950-955. doi:10.1111/ijd.2010.49.issue-8

27. Miller I, Lynggaard CD, Lophaven S, Zachariae C, Dufour DN, Jemec GB. A double-blind placebo-controlled randomized trial of adalimumab in the treatment of hidradenitis suppurativa. $\mathrm{Br}$ J Dermatol. 2011;165(2):391-398. doi:10.1111/bjd.2011.165.issue-2

28. Sotiriou E, Goussi C, Lallas A, Chovarda E, Apalla Z, Lazaridou E. Ioannides DA prospective open-label clinical trial of efficacy of the every week administration of adalimumab in the treatment of hidradenitis suppurativa. J Drugs Dermatol. 2012;11(5 Suppl):s15-s20.

29. AbbVie Ltd. A Phase 3 multicenter study of the safety and efficacy of adalimumab in subjects with moderate to severe hidradenitis suppurativa - PIONEER I. Clin Study Rep Adalimumab Protocol. 2015.

30. AbbVie Ltd. A Phase 3 multicenter study of the safety and efficacy of adalimumab in subjects with moderate to severe hidradenitis suppurativa - PIONEER II (Clinical study report (Interim), adalimumab/ protocol M11-810). 2015.

31. Jemec GBE, Okun MM, Forman SB, ET AL. Adalimumab mediumterm dosing strategy in moderate-to-severe hidradenitis suppurativa: integrated results from the phase III randomized placebo-controlled PIONEER trials. Br J Dermatol. 2019;181(5):967-975.

32. Zouboulis CC, Okun MM, Prens EP, et al. Long-term adalimumab efficacy in patients with moderate-to-severe hidradenitis suppurativa/ acne inversa: 3-year results of a phase 3 open-label extension study. J Am Acad Dermatol. 2019;80(1):60-69. doi:10.1016/j.jaad.2018. 05.040

33. Kimball AB, Tzellos T, Calimlim BM, Teixeira HD, Geng Z. Okun MMAchieving Hidradenitis suppurativa response score is associated with significant improvement in clinical and patient-reported outcomes: post hoc analysis of pooled data from PIONEER I and II. Acta Derm Venereol. 2018;98(10):932-937. doi:10.2340/000155553012 


\section{Publish your work in this journal}

Patient Related Outcome Measures is an international, peer-reviewed, open access journal focusing on treatment outcomes specifically relevant to patients. All aspects of patient care are addressed within the journal and practitioners from all disciplines are invited to submit their work as well as healthcare researchers and patient support groups.

Submit your manuscript here: http://www.dovepress.com/patient-related-outcome-measures-journal
The manuscript management system is completely online and includes a very quick and fair peer-review system. Visit http://www. dovepress.com/testimonials.php to read real quotes from published authors. 